\title{
Perception de la performance des organisations publiques locales : Une analyse du discours des citoyens-usagers des municipalités au Cameroun
}

\author{
Axel Dieudonné Mbarga, PhD \\ Enseignant au Département de Gestion Financière et Comptabilité, Ecole \\ Supérieure des Sciences Economiques et Commerciales, \\ Université de Douala, Cameroun \\ Jean Claude Mbassi, PhD \\ Enseignant au Département de Marketing et Organisation, Faculté des \\ Sciences Economiques et de Gestion Appliquée, \\ Université de Douala, Cameroun
}

Doi:10.19044/esj.2019.v15n22p116 URL:http://dx.doi.org/10.19044/esj.2019.v15n22p116

\section{Résumé}

La littérature sur l'évaluation et le pilotage de la performance dans les organisations publiques montre que les auteurs retiennent des visions partielles du concept de performance, alors que celui-ci connait des développements importants depuis des décennies. Cette recherche vise à cerner les logiques des acteurs qui sont au coeur de la gestion des collectivités locales d'une part, d'autre part décrire des représentations y afférentes que les usagers s'en font. Il s'agit de mettre en perspective les critères qu'ils prennent en compte pour apprécier la performance des municipalités. Pour y parvenir, une étude qualitative a été réalisée auprès de trente (30) citoyens-usagers des municipalités au Cameroun. A l'aide de l'analyse de contenu thématique, ce travail révèle que le concept de performance au niveau local est une notion plurielle, multidimensionnelle et contingente. Selon les citoyens, la performance est appréciée au moyen du degré de satisfaction que tirent les usagers de la consommation des biens et services sociaux de base.

Mots-clés : Performance, organisations publiques locales, citoyens/usagers, étude qualitative, Cameroun 


\title{
Perception of Local Public Organizations' Performance: An Analysis of Citizens-Users Discourse in Cameroon's Municipalities
}

\author{
Axel Dieudonné Mbarga, PhD \\ Enseignant au Département de Gestion Financière et Comptabilité, Ecole \\ Supérieure des Sciences Economiques et Commerciales, \\ Université de Douala, Cameroun \\ Jean Claude Mbassi, PhD \\ Enseignant au Département de Marketing et Organisation, Faculté des \\ Sciences Economiques et de Gestion Appliquée, \\ Université de Douala, Cameroun
}

\begin{abstract}
Literature about assessment and management of the performance in public organizations reveals that some authors' still partially address this notion, although there have been significant developments some decades ago. This paper not only aims at understanding the logics of actors who are in the forefront of the management of local authorities; but also targets the description of associated thoughts that the citizens-users may have. It is a question of bringing to light the criteria taken into account to evaluate the municipalities' performance. To this end, a qualitative survey of thirty (30) citizens-users of municipalities' in Cameroon has been carried out. Based on the thematic content analysis, this work shows that performance at the local level is a pluralistic, multidimensional and contingent concept. According to citizens, the appreciation of the performance results from the degree of satisfaction they have after using goods and social services.
\end{abstract}

Keywords: Performance, Local public organizations, Citizens/users, Qualitative study, Cameroon

\section{Introduction}

Le management public fait l'objet de commentaires contrastés. Il a pris à ce jour les tournures d'une guerre de religion (Hood, 2005). Pendant que certains auteurs prennent acte des finalités extraverties et des principes de régulation spécifique aux organisations publiques pour dénoncer l'importance en leur sein des dispositifs inventés dans le secteur privé (Gibert, 1998). D'autres auteurs à l'inverse, insistent sur l'échec de la régulation 
bureaucratique et prennent acte de la performance des organisations privées pour justifier le transfert de ces méthodes dans les organisations publiques (Chevalier, 2005). Toutefois, selon Merrien (1999) cette guerre de religion serait terminée au profit du second bloc d'auteurs. L'administration publique doit être performante, il faut désormais un Etat efficace et qui minimisera les dépenses que plusieurs qualifient de bureaucratiques (Percebois, 2006).

La performance des organisations publiques apparaît dès lors de nos jours comme un des sujets clés dans les recherches et les pratiques des administrations publiques. Pour Walker et Boyne (2009), la problématique de la performance de ces entités est une des questions qui définissent le champ du management public. Les questions essentielles concernent alors les effets de l'environnement externe, les caractéristiques organisationnelles et le management de la performance. L'importance accordée au concept de performance des organisations publiques illustre cette transformation. La performance en tant qu'idéal vers lequel l'administration tend a vu sa zone d'influence allant des services publics industriels et commerciaux vers les administrations régaliennes comme les hôpitaux, les universités ou encore les collectivités locales. Cette notion est donc le reflet de notre époque. L'on entend dire qu'il faut être "performant" dans son travail, dans ses études, dans sa vie familiale, tout ceci a donc conduit au culte de la performance. Ce concept a fait l'objet de nombreuses recherches et modes d'opérationnalisation visant à clarifier sa nature, ses dimensions, ses mesures voire ses déterminants (Sogbossi, 2010 ; Véran, 2008 ; Van der Stede et al, 2006 ; Poincelot et Wegmann, 2005).

Traditionnellement, le concept de performance est polarisé sur le résultat, mais il est apparu fortement polysémique au point qu'il intègre une certaine subjectivité (Sogbossi, 2010, Ndangwa et al, 2007). Les questions d'évaluation de la performance ont alors conduit à un glissement passant des entreprises privées vers les administrations publiques. A ce sujet, Bourguignon (1997) rappelle que ce terme, avant d'être adapté pour les entreprises, s'utilisait initialement dans deux domaines particuliers notamment le sport et la mécanique. Dès lors, la vision traditionnelle de son évaluation essentiellement focalisée sur les critères d'efficacité a progressivement été remise en cause, et une vision multidimensionnelle a été développée. La performance ne se limite plus alors à l'atteinte des objectifs économiques et financiers, mais s'étend de plus en plus à la satisfaction des préoccupations sociétales notamment sociales et environnementales (Reynaud, 2003). A ce sujet, Saulquin et Schier (2005, p.7) indiquent que la notion de performance intègre par définition une certaine « subjectivité », dès lors, «la performance a autant de facettes qu'il existe d'observateurs à l'intérieur et à l'extérieur de l'organisation. Elle est ainsi définie par ceux qui vont utiliser l'information. Elle n'a d'importance (de valeur) que par rapport 
à ce que l'utilisateur de cette information va en faire ». Les deux auteurs ajoutent que la performance reste «une affaire de perception » entre les individus, ce qui risque d'engendrer des conflits dans des périodes de crises en raison de la divergence de cette perception. Ainsi, ils concluent que : «Le concept (de performance) possède autant de significations qu'il existe d'individus ou de groupes qui l'utilisent. Pour un dirigeant, la performance pourra être la rentabilité ou la compétitivité de son entreprise; pour un employé, elle pourra être le climat de travail ; et pour un client, la qualité des services rendus. La multiplicité des approches possibles en fait un concept surdéterminé, et curieusement, il demeure indéterminé en raison de la diversité des groupes qui composent l'organisation». Au demeurant, la performance est fortement une affaire de perception entre les individus et cette perception peut changer selon les zones culturelles (Olomo, 1987).

Le présent travail qui se situe dans cette approche perceptuelle a pour objectif de décrire et d'analyser la perception qu'ont les acteurs impliqués dans la gestion des collectivités locales de la notion de performance particulièrement au Cameroun. Il s'agit ici de faire ressortir les catégories importantes à prendre en compte dans l'évaluation de la performance des collectivités locales. Pour y parvenir, cinq points sont présentés. Après les propos introductifs (1), les contours de la littérature relative aux considérations théoriques du concept de performance sont abordés (2), ensuite les choix méthodologiques effectués (3), une exposition des résultats (4) et enfin la discussion des résultats est effectuée tout en tirant les conclusions et les contributions de la recherche(5).

\section{Revue de la littérature}

Pour toute recherche sur la notion multidimensionnelle de performance, des clarifications et précisions s'avèrent nécessaires tant sur sa définition et sa mesure en contrôle de gestion que sur son glissement dans la sphère des organisations publiques.

\section{Définition et mesure du concept polysémique de performance}

Le terme de «performance » a suscité de larges débats dans la littérature en contrôle de gestion. Cela peut être dû à l'ambigüité et à la diversité des sens qu'on peut lui donner selon le contexte. Dans l'acception commune, la performance désigne un «exploit», un «succès». Être performant c'est être compétitif, c'est atteindre des résultats supérieurs à d'autres personnes, d'autres individus par rapport à des objectifs préétablis.

Bouquin (2004) rappelle qu'en français, le mot désigne l'idée d'une réalisation d'exception, alors qu'il est utilisé en contrôle de gestion qu'en référence à son sens en anglais pour désigner un résultat. Ce résultat sert en premier lieu à comparer les réalisations aux attentes ou prévisions. Récemment, cette notion a fait l'objet de nouveaux débats sur sa définition. 
Deux visions différentes sont ainsi développées. La première critique l'utilisation de ce terme qui était uniquement relatif aux indicateurs de coûts et de rentabilité à court terme en militant en faveur de l'introduction d'autres mesures de fonctionnement. La deuxième critique voulait «mettre en avant une version plus actualisée de ce que l'on nommait l'excellence dans les années $1980 \gg$ (Bouquin, 2004, p.62), et revenir ainsi à la vieille connotation française de la performance. Le contrôle de gestion considère la performance comme «l'impact qu'a une activité, un centre de responsabilité, un produit, etc., sur la performance globale de l'entreprise »(Bouquin, 2004, p.62). Il propose une représentation détaillée de la performance, comme un processus, décomposé en trois éléments : l'économie, l'efficience et l'efficacité.

Bouquin définit ensuite les éléments de ce processus comme suit : l'économie consiste à se procurer les ressources au moindre coût ; l'efficience consiste en la maximisation de la quantité obtenue de produits ou de services à partir d'une quantité donnée de ressources. L'efficacité est le fait de réaliser les objectifs et finalités poursuivis. Mesurer la performance revient donc à mesurer les trois dimensions qui la composent.

Cependant, d'autres définitions du concept en contrôle de gestion ont vu le jour. Bourguignon (1997, p.934) propose par exemple une définition du terme performance en contrôle, et le considère «comme la réalisation des objectifs organisationnels quelles que soient la nature et la variété de ces objectifs. Cette réalisation peut se comprendre au sens strict (résultat, aboutissement), ou au sens large d'un processus qui mène au résultat (action) ».

Le concept est donc polysémique, même en contrôle de gestion. Burlaud et Simon (2006) montrent que la vision de la performance chez les contrôleurs de gestion a évolué avec le temps, elle est alors au cœur des processus dudit contrôle. Elle renvoie implicitement aux hypothèses et aux préceptes du contrôle de gestion, notamment celles de l'évaluation et de la réalisation d'une action préétablie. Cette action sera évaluée en fonction des objectifs qui lui étaient assignés. Cette acception amène à considérer qu'il n'existe point de performance si l'on ne définit pas, au préalable des objectifs chiffrés et des ressources employées. Les ressources consommées représentent le «coût» de l'action et la «valeur» renvoie à la satisfaction des besoins sociaux. Dans cette perspective, Lorino (2003, p.11) définit la performance comme «le déploiement du couple valeur-coût dans les activités de l'organisation ».

Les organisations publiques déploient le plus souvent d'énormes ressources pour l'atteinte des objectifs en vue de la création de la valeur publique. Dès lors, l'évaluation de la performance au sein des entités publiques devient une nécessité. 


\section{Intégration du management de la performance dans les collectivités locales}

Deux types d'arguments sont généralement avancés pour justifier le recours à la mesure de la performance dans le secteur public (De Bruijn, 2002 ; Behn, 2003). D’abord les qualités génériques des systèmes de mesure et de pilotage de la performance (SMPP), ensuite les avantages spécifiques ou particuliers des SMPP dans le secteur public. Selon Osborne et Gaebler (1992), les bénéfices importants provoqués par la mesure de la performance dans le secteur privé peuvent aisément être obtenus dans le secteur public. Par conséquent, les auteurs prolongent ce que Greiling (2006, p.1) surnomme le 'mentra' d'Osborne et Gaebler repris par Guenoun (2009, p.199) présenté en ces termes :

- $\quad$ Si on ne mesure pas ses résultats, il est impossible de distinguer un succès d'un échec.

- Si vous ne pouvez identifier un succès, vous ne pouvez pas le récompenser.

- Si vous ne pouvez récompenser un succès, vous récompensez très probablement un échec.

- Si vous ne pouvez identifier un échec, vous ne pouvez pas le corriger.

- Si vous pouvez présenter vos résultats, vous pouvez conquérir l'adhésion des usagers.

Cette conception est alors assez proche du modèle développé par Bouckaert et Halligan (2008) qualifié de modèle rationnel de la décision pour qui, la transparence interne accrue apportée par les SMPP est à la source de toutes les améliorations organisationnelles. La justification de l'utilisation des indicateurs de performance dans le secteur public est donc manifeste par un constat simple que les organisations privées utilisent des indicateurs de performance et sont plus efficientes que les organisations publiques (Behn, 2003). Cette mesure de la performance est censée favoriser l'autonomie de l'organisation (De Bruijn, 2002).

D'autres justifications de l'intérêt de la mesure de la performance des entités publiques évoquent des avantages spécifiques et particuliers liés au secteur public lui-même. Pour Rochet (2004) et Mongbe (2007), l'argument le plus fondamental renvoie à la dimension démocratique de la mesure de la performance. Il s'agit en effet d'un côté, de rendre le pouvoir aux représentants des citoyens en renforçant le pouvoir du parlement en matière budgétaire et d'un autre côté, de mettre sous pression les organisations publiques, et de donner un droit à l'information aux citoyens (Bouckaert, 2003) ${ }^{7}$. Aussi, dans

${ }^{7}$ En effet, une série de dispositions intégrées dans les réformes budgétaires notamment : comptabilité par destination, publication de rapports d'activités librement consultables est censée améliorer la lisibilité des budgets, l'information du citoyen et la transparence des organisations publiques. 
une perspective plus instrumentale, la mesure de la performance est-elle censée améliorer les choix des consommateurs de services publics, surtout en matière de choix des services publics. La mesure de la performance permettra donc une sorte de comparaison des services entre eux et conduira les usagers à des choix éclairés (Talbot, 2005, p.498). Selon Emery (2006), un autre argument en faveur du développement du management de la performance dans le secteur public découle du paradigme de la qualité ${ }^{8}$. Dans cette logique, les organisations publiques confrontées aux usagers de plus en plus exigeants et constitués en associations de défense de leurs droits (consuméristes), doivent s'engager sur un niveau de qualité de service délivré.

Sur la base des précédents arguments, l'intérêt de la mise en exergue de la mesure de la performance au sein des organisations publiques n'est plus à démontrer. Cependant, une adaptation de la conception de la performance au sein des municipalités est souhaitable.

\section{Les débats théoriques et controversés du management de la performance dans les organisations publiques}

En effet, la problématique de la performance des organisations publiques est une des questions qui définissent le champ du management public en ce début du $21^{\text {ème }}$ siècle (Walker et Boyne, 2009). La performance est donc l'objectif central des réformes administratives menées partout dans le monde (Van Dooren, 2006, cité par Guenoun et Saléry, 2009, p.893). Les questions essentielles concernent alors les effets de l'environnement externe, des caractéristiques organisationnelles et même du management de la performance. Ce concept fourre-tout, loin d'être neutre, accompagne la diffusion à l'échelle planétaire du corpus anglosaxon du New Public Management (Boyne, 1996 ; Guenoun et Saléry, 2009), où les idées forces consistent à focaliser l'attention sur les outils et instruments de gestion. En matière de mesure de la performance, le modèle du NPM en ce qui concerne le rapport Etat-collectivités locales se caractérise par une politique d'imposition de référentiels de mesure de la performance au sein des entités locales par 1'Etat (Boyne, 2002 ; Padovani et Scorsone, 2009) ${ }^{9}$. Talbot (2006)

${ }^{8}$ Pour Bouckaert (2006: 12), la qualité est aussi bien une dimension importante de la performance publique qu'un moteur du développement des systèmes de mesure de la performance.

${ }^{9}$ Les auteurs ont proposé un cadre d'analyse visant à permettre la prise en compte du contexte de la mesure de la performance composé de trois dimensions ; le régime de la performance qui concerne la structure et l'influence des organismes de normalisation qui imprègnent les systèmes de mesure de la performance développés au niveau des gouvernements locaux. La nature des relations intergouvernementales qui suppose d'identifier la manière dont les gouvernements locaux, nationaux et autres interagissent et l'axe mesure de la performance qui s'organise selon la logique retenue par le gouvernement dans le contrôle de sa performance et l'atteinte des objectifs stratégiques. 
a plutôt montré à l'inverse une simple relation hiérarchique 'principal/agent" , entre les gouvernements. Selon lui, il existe une multitude de principaux (services gouvernementaux, ministères fonctionnels, parlements, organes d'audit, organes d'inspection et de régulation, organes judiciaires, institutions professionnelles et usagers, etc.) qui peuvent être associés à la définition du contexte dans lequel un gouvernement "agent" s'engage dans le développement et l'adoption d'un système de mesure de la performance. Chacun de ces acteurs peut être habilité à discuter et à imposer l'utilisation des mesures de performance pour l'ensemble du gouvernement local ou alors pour certaines de ses compétences (Talbot, 2003). Cette analyse reste cependant sommaire dans le contenu et peut être complétée à l'aide du cadre proposé par Bouckaert et Halligan (2008) pour qui, le management n'est pas qu'une affaire de mesure. Ils vont donc proposer une approche plus systémique de la performance et définir le management de la performance comme l'articulation de trois séries d'activités : la mesure de la performance, l'incorporation des mesures de la performance et l'utilisation des informations produites. Guenoun et Saléry (2009, p.895) en joignant de multiples travaux notamment de Padovani et Scorsone (2009), de Bouckaert et Halligan (2008); obtiennent un cadre à la fois contextuel, descriptif et dynamique du management de la performance publique locale.

De nombreux modèles de gestion de la performance publique inspirés des théories de gestion de la performance des entités privées ont été développés (Hood, 1995 ; Pollit et Bouckaert, 2004). Cependant, compte tenu des spécificités du secteur public caractérisé par la double fonction de production et de l'aspect multidimensionnel des finalités poursuivies, des ajustements s'imposent quant à la mesure de la performance (Carassus et al., 2012, p.4 ; Carassus et al., 2014). La performance dans ce cadre se définit alors par des indicateurs multi-critères et multi-acteurs (Dohou et Berland, 2007, p.10). Elle se fonde à la fois sur les indicateurs financiers et non financiers (Reynaud, 2003, p.10 ; Capron et Quairel-Lanoizelée, 2006 ; Baret, 2006, p.2 ; Saulquin et Schier, 2005). Les réflexions sur les indicateurs non financiers seront alors présentées dans le Balanced ScoreCard (BSC) de Kaplan et Norton (1992, 1996, 2001). Il y a donc une évolution de la performance des aspects financiers mesurés pour les actionnaires (shareholders) au moyen de la théorie de l'agence (Jensen et Meckling, 1976) vers la performance mesurée pour les stakeholders au moyen de la théorie des parties prenantes (Freeman, 1984 ; Wood et al., 2018).

\section{Le Balanced ScoreCard comme modèle d'évaluation de la performance dans les collectivités locales}

Le BSC est une combinaison de mesures financières et opérationnelles classées selon quatre axes notamment : les résultats financiers, la satisfaction 
des clients, les processus internes et l'apprentissage organisationnel. Dans la conception du BSC, l'axe apprentissage organisationnel permet de développer les compétences humaines qui induisent une amélioration de la productivité et la qualité des services (axe processus internes), ce qui va contribuer à la plus grande satisfaction des clients (axes clients) et servir au final les objectifs financiers de l'organisation (axe financier). La principale limite formulée visà-vis du BSC est qu'il reste orienté sur le résultat économique, financier et ne permet pas véritablement une évaluation de la performance non financière plutôt mesurée dans le cadre des organisations publiques. Le BSC va donc faire l'objet de nombreuses recherches et progressions en vue de conduire à une meilleure évaluation de la performance (Germain et Gates, 2007).

Kaplan et Norton ont développé en 1992 le BSC classique dont le rôle ne s'est pas limité à la mesure de la performance, mais au pilotage stratégique. Tour à tour, ils ont développé le BSC prospectif en 1996 et le BSC évolué de 2001 qui intègrent la capacité de la firme à être citoyenne. En considérant que la firme doit faire partie intégrante de la mesure de la performance, ceci sera matérialisé par les indicateurs sociétaux sur l'axe "Processus internes" et par l'extension de l'axe "Clients" à tous les partenaires de l'entreprise. De nombreuses recherches vont donc tenter de prolonger les travaux de Kaplan et Norton et dans cette perspective, Hockerts (2001) proposera le " Sustainability $B S C$ ", Bieker (2002) fera passer cet outil de quatre à cinq axes avec l'introduction de l'axe "Sociétée". Par la suite, Supizet (2002) suggère le "Total BSC' qui suppose six relations causales entre les parties prenantes notamment : les actionnaires, les clients, les usagers de l'entreprise en tant que personne morale, les partenaires, le personnel et la collectivité. Malgré ces évolutions, la mesure d'une performance globale reste assez préoccupante. Cette problématique est encore plus préoccupante lorsqu'il s'agit de l'évaluation des performances des organisations publiques comme les communes.

Les particularités du BSC dans l'évaluation de la performance semblent le rendre plus compatible avec les spécificités du secteur public. Compte tenu de ce que les organisations publiques ne recherchent pas principalement la rentabilité des capitaux investis, l'articulation entre indicateurs financiers et non financiers mise en exergue dans le BSC le rend plus apte à la mesure de la performance dans les structures publiques locales. A cet effet, quelques auteurs ont tenté des adaptations du BSC au secteur public (Aidemark, 2001 ; Mc Kendrick et Hastings, 2002 ; Moullin, 2006 ; Farneti, 2006 ; Chauvey, 2006 ; Chapet, 2007). Dans cette perspective, Moullin (2002, p.6) va développer le "Public Sector ScoreCard" censé améliorer la qualité de service. L'auteur va donc proposer d'ajouter une dimension nouvelle d'évaluation de la performance en vue d'apprécier la satisfaction des usagers/consommateurs de services publics. Cet axe sera 
qualifié d'axe “Usagers/Parties Prenantes". Le PSS aménagé qui comporte alors cinq axes sera appliqué dans certaines entités du secteur hospitalier, des transports et dans une collectivité locale britannique (Moullin, 2006). Chapet (2007) propose lui aussi cinq axes ${ }^{10}$ et recommande d'identifier une partie prenante pour chacun des axes car, cette mise en cohérence axe de performance/partie prenante est censée favoriser l'alignement de ces axes sur les attentes et aspirations des parties prenantes. Farneti (2006) va développer un BSC pour les collectivités locales italiennes et proposera de remplacer l'axe "Clients" de Kaplan et Norton par l'axe "Citoyens". Au final, il est assez difficile d'apprécier la performance dans les organisations publiques locales en termes de part de marché, de résultat économique ou financier, car les résultats dans les municipalités paraissent intangibles et diffus.

L'Etat a donc su se doter depuis toujours d'indicateurs de mesure de la performance des affaires publiques (Khulmann, 2010, p.3). Kaufmann et al. (2005) vont tenter de déterminer les facteurs clés de la performance des villes. La performance des villes a été appréciée par l'accès et la qualité des services publics délivrés. Les auteurs mettent donc en évidence une relation non linéaire entre la taille, le type de ville et la performance. Les très grandes villes (les villes capitales et les villes portuaires) apparaissent potentiellement peu performantes car elles offrent difficilement un bon accès aux services tels que la gestion des égouts, l'accès à l'eau potable, à l'électricité, au téléphone et la qualité des infrastructures de base (Kaufmann et al., 2005, p.37).

En revenant, dans le contexte du Cameroun, il faut relever qu'il existe très peu d'études sur les municipalités. Avelé et Bikourane (2016), Avelé et Nyengué Edimo (2015), Avelé (2013a ; 2013b ; 2013c) dans la perspective de recherche sur les effets des outils de contrôle de gestion sur la performance des services publics locaux démontrent qu'il existe un lien entre les facteurs de contingence structurelle et comportementale et la performance des municipalités camerounaises. Les auteurs attestent qu'il existe un lien positif entre la participation budgétaire et la performance des services publics municipaux. De plus, ils observent que : «plus la structure municipale est décentralisée, mieux l'on réalise les objectifs de performance de l'institution communale ». Ce résultat rejoint les hypothèses de la théorie des choix publics qui suggèrent que la performance publique est élevée si les administrations centrales sont déconcentrées et fragmentées. Selon cette théorie, les grandes organisations centrales sont par essence insensibles et irresponsables aux besoins et attentes de leur public, elles sont alors inefficaces et échouent considérablement à l'atteinte de leurs objectifs formels. Dans le même sens, Avele (2013c), Avelé et Bikourane (2016), Avelé et Nyengué Edimo (2015)

10 Il distingue les axes suivants: finalités, réalisations, processus, contributions et compétences. 
obtiennent des résultats proches de ceux de Boyne (1996) et de Gray et Jenkins (1995). Boyne (1996) à partir des données sur six collectivités locales va tester l'hypothèse du NPM selon laquelle il existe une relation négative entre l'échelle du service public et la performance. Les auteurs montrent que les plus petites unités publiques locales ou décentralisées sont plus performantes. Quant à Gray et Jenkins (1995, p.86), les nouvelles théories du management public, dans leur unicité attaquent le modèle bureaucratique traditionnel de la structure administrative en préconisant l'efficacité à travers les formes de décentralisation qui permettent une évaluation plus large de la performance. Sur la base de ces développements, la principale proposition de ce travail est que : "La performance dans les collectivités locales est une réalité plurielle, multidimensionnelle et contingente en contexte du Cameroun ».

\section{Méthodologie adoptée pour l'évaluation de la performance des municipalités}

Ce point ambitionne de justifier le choix de l'étude qualitative et de préciser dès lors les outils et techniques retenus pour le recueil et l'analyse des informations dans le cadre de cette recherche. A partir des entretiens effectués auprès des représentants des citoyens/usagers (les chefs de quartier, chefs de bloc et chefs de village), cette étude vise à enrichir la construction du concept de performance des institutions communales.

Des entretiens ont été réalisés auprès des acteurs impliqués dans la gestion des municipalités principalement les représentants des citoyens/administrés en tant qu'auxiliaires des conseils municipaux et porteparole des populations. De plus, ces autorités traditionnelles sont au cœur de la régulation de la société dans leur territoire géographique de compétence. Il s'est agi d'aller vers ces personnalités qui connaissent mieux les attentes, besoins et aspirations des populations à la base afin de recueillir leurs avis sur la conception qu'ils se font du concept de performance d'une collectivité locale. Les personnes interrogées sont issues des collectivités locales des régions du Centre et du Littoral, Yaoundé et Douala. Au final, 30 personnes ont été interviewées dont quatre femmes et vingt-six hommes. Par ailleurs, le principe de saturation sémantique a été retenu quant au choix du nombre de personnes à interviewer (Romelaer, 2005). ces entretiens ont été effectués par les auteurs et en vue de préserver l'anonymat, les noms et fonctions des personnes interviewées n'ont pas été mentionnés. Toutes les informations ont été collectées au moyen d'un guide d'entretien ${ }^{11}$ déposé à l'avance auprès des interviewés et des entretiens semi-directifs d'une durée moyenne d'environ 50 minutes, d'un minimum de 35 minutes et d'un maximum de 2 heures environ ont été administrés.

\footnotetext{
${ }^{11}$ Les entretiens ont été effectués sur la base d'un guide d'entretien qui est consigné en annexe.
} 
Enfin, le logiciel NVivo a été choisi en ce qu'il permet l'analyse des données à travers un système de classification et de rangement de l'information. D'où, une analyse de contenu thématique a été effectuée en ce sens qu'elle permet d'aller au-delà de la simple prise en compte de l'occurrence mais bien plus en privilégiant «la valeur du thème » en vue de souligner son importance (Ben Zekri et Zaiem, 2016). La perception de la performance par les administrés va conduire à une certaine représentation de la réalité au moyen des éléments tangibles ou intangibles susceptibles d'entrainer la formalisation d'une grille d'évaluation des performances des collectivités locales dans le contexte du Cameroun.

\section{Résultats}

L'objectif poursuivi ici est de mettre en exergue la perception qu'ont les interviewés du concept de performance dans les collectivités locales. L'analyse de contenu thématique des résultats issus des entretiens semidirectifs montre que la performance dans l'ensemble, est appréciée en termes de satisfaction des attentes des populations par la mise à leur disposition des infrastructures susceptibles d'améliorer la qualité de la vie. Dans cette veine, les codes de perception de la performance ont été mis en exergue dont la synthèse est proposée dans le tableau ci-dessous :

Tableau 1: synthèse des résultats de l'étude qualitative

\begin{tabular}{l|c|c|c}
\hline Catégories & $\begin{array}{c}\text { Nombre de } \\
\text { citations }\end{array}$ & $\begin{array}{c}\% \text { de } \\
\text { citations }\end{array}$ & $\begin{array}{c}\text { Nombre } \\
\text { d'entretiens }\end{array}$ \\
\hline Routes & 67 & $23,59 \%$ & 30 \\
\hline Electrification & 48 & $16,90 \%$ & 30 \\
\hline Adduction en eau & 43 & $15,14 \%$ & 30 \\
\hline Salubrité (bacs à ordures, toilettes, etc.) & 22 & $07,75 \%$ & 17 \\
\hline Etat-civil & 19 & $06,69 \%$ & 15 \\
\hline Ponts & 16 & $05,63 \%$ & 13 \\
\hline Ecoles & 15 & $05,28 \%$ & 15 \\
\hline Hôpitaux & 13 & $04,58 \%$ & 09 \\
\hline Hôtels de ville & 08 & $02,82 \%$ & 08 \\
\hline Espaces de loisir & 06 & $02,11 \%$ & 05 \\
\hline Signature des documents & 05 & $01,76 \%$ & 03 \\
\hline Aides sociales (handicapés et nécessiteux) & 04 & $01,41 \%$ & 02 \\
\hline Partenaires externes au financement & 04 & $01,41 \%$ & 03 \\
\hline Marchés & 04 & $01,41 \%$ & 04 \\
\hline Finances propres à la commune & 03 & $01,06 \%$ & 01 \\
\hline Funérarium cimetière & 03 & $01,06 \%$ & 02 \\
\hline Emprunts externes & 02 & $00,70 \%$ & 02 \\
\hline Parcs d'engins & 02 & $00,70 \%$ & 01 \\
\hline Total de la catégorie & 284 & $100,00 \%$ & III \\
\hline
\end{tabular}

Source : entretiens avec les représentants des populations locales 


\section{Discussions}

Les résultats consignés dans le tableau 1 présentent la réalité contextuelle des préoccupations des acteurs impliqués dans le management des collectivités locales qui définissent la performance d'une municipalité en termes de satisfaction des citoyens/usagers désormais clients. L'on peut alors relever que pour les bénéficiaires des services publics municipaux, la performance combine les éléments tangibles à ceux non tangibles et l'offre en infrastructures sociaux de base est essentielle en matière d'évaluation de la performance. La performance est définie à partir d'un certain nombre d'indicateurs regroupés en plusieurs dimensions. Ces populations, en tant que partie prenante influente (Wood et al., 2018) dans la gestion de l'institution communale font ressortir le caractère pluriel du concept. La performance des collectivités locales renvoie alors aux infrastructures de base, à la capacité de la commune à mobiliser les ressources financières et à la qualité du service public.

\section{Offre et accès aux infrastructures sociales de base comme dimension tangible de la performance}

Le tableau 1 fait ressortir une multitude de critères de mesure de la performance par les citoyens-usagers des municipalités. L'analyse des fréquences d'apparition des thèmes révèle que douze des dix-huit critères d'évaluation de la performance renvoient à la satisfaction des populations en matière d'offre en infrastructures de base. Les citoyens-clients interviewés reconnaissent tous la nécessité des infrastructures telles que; les routes, l'électrification et l'accessibilité à l'eau potable qui obtiennent une forte occurrence dans le discours de tous les interviewés. De plus, ils relèvent à côté de ces services prioritaires l'importance pour les dirigeants communaux à se pencher résolument sur les questions de salubrité, des ponts, des écoles et des hôpitaux pour ne citer que ceux-là. Les quatre premiers codes (routes, électrification, eau et salubrité) obtiennent 180 citations environ $65 \%$ des citations totales relevées dans le discours des interviewés. C'est dire que, l'accroissement et l'amélioration de l'offre en infrastructures sociales par les communes est gage de la plus grande satisfaction des populations car ces biens agissent sur la qualité de la vie en matière d'éducation, de santé, de communication, etc. Dès lors, une commune qui offre des infrastructures de qualité à ses populations locales est perçue comme étant performante. Cet état de choses transparaît également dans le discours de certains interviewés: «Oui il y a par exemple le maire qui ouvre pour l'éclairage public en distribuant les lampadaires » (Entretien, 18). «Dès qu'on a l'électricité, nous avons la sécurité »(Entretien, 7). «Lorsque les routes sont reprofilées, nous savons que nos enfants quand ils vont à l'école ne seront pas emportées par les eaux» (Entretien, 22). «A présent notre maire est à New-York pour 
défendre un projet de construction d'un funérarium, ce qui va éviter les multiples occupations des emprises sur la voie publique pour deuil» (Entretien, 15). Au demeurant, quoique la dimension infrastructurelle soit très importante dans l'évaluation de la performance des collectivités locales ici appréciée en termes non financiers, il ressort du discours des acteurs qu'il existe d'autres dimensions qui contribuent à enrichir le concept étudié.

\section{La dimension ressources financières dans l'évaluation de la performance des municipalités au Cameroun}

Les résultats révèlent que plusieurs autres facteurs ou critères intangibles expliquent la performance perçue des municipalités. En complément à la première dimension, l'observation de la deuxième dimension permet de faire ressortir les différentes sources de financement auxquelles les responsables communaux recourent pour la réalisation des investissements sociaux. Ainsi, même si les citoyens-clients reconnaissent les difficultés auxquelles font face les managers municipaux, il existe tout de même des mécanismes auxquels ils peuvent recourir en vue d'accroître l'offre en infrastructure pour une plus grande satisfaction des populations. Trois éléments permettent de définir cette dimension notamment ; le recours aux partenaires et particulièrement à la coopération décentralisée, les financements propres de la commune au moyen de la fiscalité locale et les emprunts externes. Toutefois, le caractère marginal de cette dimension est mis en perspective avec seulement 9 références sur 284 pour $3,16 \%$, ce qui est faible mais susceptible d'avoir un effet indirect sur la satisfaction des administrés par ailleurs électeurs. C'est dire que cette facette de la performance est aussi importante car sans ressources, aucun projet de développement ne pourrait être initié et conduit à terme dans les communes. "Par la coopération décentralisée, les maires peuvent aussi arriver à faire les dons dans les centres de santé, et écoles ici chez nous, il y a aussi les handicapés. Et même alors comment les autres communes font notre maire doit aussi faire le jumelage avec les mairies étrangères qui accompagnent beaucoup de projets ailleurs non; pourquoi pas nous ?» (Entretien, 15).

L'accès aux ressources financières est une préoccupation pour les citoyens qui sont de plus en plus exigeant sur la quantité et la qualité des services qui sont offerts par les municipalités. Mais ils reconnaissent que si les communes mobilisent davantage de ressources, elles pourraient améliorer l'offre des services sociaux et la satisfaction des populations.

\section{La qualité de service public : un attribut intangible de la performance des municipalités au Cameroun.}

La dernière dimension évoquée par les interviewées renvoie aux facteurs non tangibles de la performance liés à la qualité de service public. Elle apparaît 
décomposée en trois sous catégories ; les services liés à l'état-civil avec 19 citations chez 15 interviewées, la signature des documents dans les mairies (5 références) et l'assistance sociale au moyen des services susceptibles d'améliorer le cadre de vie des citoyens (4 références). La multiplicité des objectifs poursuivis par les collectivités locales ne les met pas dans les contraintes fortes d'évaluation de la performance sous l'angle financier comme c'est très souvent le cas dans les entreprises privées. Les citoyens reconnaissent la grande importance du service d'état-civil pour une mairie en tant que critère d'évaluation de la performance non financière de ces entités. Cet état de choses provient du discours des interviewés "Il y a d'abord les actes d'état-civil parce que la vocation d'un maire c'est de concilier les éléments de la vie ; la naissance, le mariage et le décès et notre service le plus sollicité et le plus fréquenté est l'état-civil »(Entretien, 11). "Il y a beaucoup de choses à faire par exemple on nous a demandé de répertorier les enfants vulnérables et les orphelins du SIDA, on leur a dit vous irez chercher les dons chez le chef et ce n'est jamais arrivé » (Entretien, 3).

$\mathrm{Au}$ demeurant, il ressort que la performance des municipalités dans le contexte d'étude est un concept multi-facettes que l'on pourrait évaluer à partir de trois dimensions en vue d'accroître la satisfaction des principaux bénéficiaires des services publics locaux.

\section{Conclusion}

La principale réflexion dans la présente recherche a consisté à s'interroger sur les indicateurs de mesure et d'évaluation de la performance des organisations publiques locales. La performance dans ce cadre ne dépend pas uniquement des facteurs matériels et tangibles. Cette recherche a mis en exergue les dimensions non financières de la performance. Elle est relative à la réalisation des objectifs au plan des investissements en infrastructures, de financement par la capacité de la commune à mobiliser les ressources financières et en termes de qualité du service public local.

Les analyses de l'étude qualitative ont permis de faire émerger la perception que se font les citoyens/usagers du concept de performance. Il en résulte que cette notion fortement polysémique préserve dans le cadre de cette recherche son caractère multidimensionnel et contingent. Dès lors, elle vient approfondir les connaissances au sujet de la performance et y apporte un éclairage nouveau. Elle contribue à ce débat en permettant d'aller au-delà de l'unique indicateur synthétique d'atteinte des objectifs de performance de la commune comme le proposent les travaux d'Avelé (2013a, 2013b, 2013c). De plus, elle se situe dans une approche non pas sectorielle mais globale en ce qu'à la différence de Sotamenou (2010) qui évalue la performance des collectivités locales en ce qui concerne la gestion des ordures, la présente recherche s'intéresse à l'ensemble des activités de l'institution communale. 
En ce sens, elle fournit trois indicateurs spécifiques, contextuels d'analyse et d'évaluation de la performance des communes. La principale contribution théorique de ce travail réside alors sur le fait qu'il propose une nouvelle grille de mesure et d'évaluation de la performance des communes camerounaises sur la base de trois dimensions: la dimension d'atteinte des objectifs $d$ 'investissement en infrastructures de base, celle de financement au moyen de la mobilisation des ressources ou de leur allocation et la dimension de la réalisation des objectifs de service public.

Par ailleurs, la principale faiblesse de ce travail réside sur son caractère exploratoire, ce qui exige une certaine réserve quant à la mobilisation des informations que dans leur interprétation. De plus, pour apprécier le niveau de performance des collectivités locales, une étude à partir des données observées sur une période plus longue et sur un nombre encore plus élevé de communes pourrait donner plus de crédibilité à la présente recherche.

\section{References:}

1. Avelé, D. (2013a). How to Reconcile Management Accounting and the Municipal Public Services of African Cities? an Empirical Study. Academy of Accounting and Financial Studies Journal, 17 (1), 1-24.

2. Avelé, D. (2013b). Reconciling Cost Calculations and Management Control Tools in Municipal Services: an Empirical Study. Academy of Accounting and Financial Studies Journal, 17 (2), 109-125.

3. Avelé, D. (2013c). The Contingency Determinants in the Performance African Municipal Public Services: an Empirical Study. African Journal of Accounting, Auditing and Finance, 2 (1), 52-64.

4. Avelé, D. \& Nyengue Edimo, P. (2015). Toward A Contingent Approach to Budget Participation and Performance of Municipal Services. International Journal of Economics and Accounting, 6 (3), 195-216.

5. Avelé, D., \& Bikourane, N. (2016). Contribution to the Knowledge of Contingent Factors on the Performance of Municipal Services. Journal of International Business Research, 15 (1), 1-14.

6. Baret, P. (2006). L'évaluation contingente de la performance globale des entreprises: une méthode pour fonder un management sociétalement responsable? IIe journée de recherche du CEROS, 1-24.

7. Behn, R. (2003). Why Measure Performance? Different Purposes Require Different Measures. Public Administration Review, 63 (5), 586-606.

8. Ben Zekri, I., \& Zaiem, I. (2016). Le marketing des ressources humaines : l'empathie et son influence sur la satisfaction au travail : cas des SSII tunisiennes. International Journal of Economics and Strategic Management of Business Process, 6, 1-7. 
9. Bieker, T. (2002). Managing corporate sustainability with the Balanced Scorecard: Developing a Balanced Scorecard for Integrity Management. Oikos PhD summer academy.

10. Bouckaert, G. (2003). La réforme de la gestion publique change-t-elle les systèmes administratifs? Revue Française d'Administration Publique, 1-2 (105-106), 39-54.

11. Bouckaert, G. (2006). Un nouvel examen de la mesure de la performance dans le secteur public. Télescope, 12 (3), 12-25.

12. Bouckaert, G., \& Haligan, J. (2008). Managing performance. International comparisons. Routledge, London.

13. Bouquin, H. (2004). Le contrôle de gestion. PUF, Gème édition.

14. Bourguignon, A. (1997). Sous les pavés, la plage... ou les multiples fonctions du vocabulaire comptable : exemple de la performance. Comptabilité, Contrôle, Audit, 1, 89-101.

15. Boyne, G. A. (1996). The intellectual crisis in British public administration: is public management the problem or the solution? Public Administration, 74, 679-94.

16. Boyne, G. A. (2002). Concepts and Indicators of Local Authority Performance: an Evaluation of the Statutory Frameworks in England and Wales. Public Money and Management, 22 (2), 17-24.

17. Burlaud, A., \& Simon, C. (2006). Le contrôle de gestion. La Découverte, Paris.

18. Capron, M., \& Qairel-Lanoizelee, F. (2006). Evaluer les stratégies de développement durable des entreprises: l'utopie mobilisatrice de la performance globale. Revue de l'Organisation Responsable, (1), 5-17.

19. Carassus, D., Favoreu C., Gardey D., \& Maurel, C. (2012). La qualification des pratiques publiques de gestion de la performance publique dans le contexte local français: un modèle plus administratif que politique. Les"démarches"locales"de"performance Cahier de recherche, 1-25.

20. Carassus, D., Favoreu C., Gardey D., \& Maurel, C. (2014). Les déterminants de l'adoption et de la mise en œuvre d'un management par la performance: application aux collectivités locales françaises. Finance Contrôle Stratégie, 17 (1), 1-24.

21. Chapet, J-M. (2007). Le système de gestion des collectivités territoriales: entre performance et délibération. Politique et Management Publics, 25 (4), 1-21.

22. Chauvey, J-N. (2006). L'intérêt du Balanced Scorecard dans l'évolution des modes de contrôle et évaluation des départements français. Politique et Management Publics, 24 (2), 69-90.

23. Chevalier, J. (2005). La reconfiguration de l'Administration Centrale. Revue Française d'Administration Publique, (116), 715-725. 
24. De Bruijn, H. (2002). Performance Measurement in the Public Sector. Strategies to Cope With the Risks of Performance Measurement. International Journal of Public Sector Management, 15 (6-7), 578594.

25. Dohou, R. A., \& Berland, N. (2007). Mesure de la performance globale des entreprises. Comptabilité et Environnement, cd-rom.

26. Emery, Y. (2006). La gestion par les résultats dans les organisations publiques: de l'idée aux défis de la réalisation. Télescope, 12 (3), 1-12.

27. Farneti, F. (2006). Balanced Scorecard Implementation in an Italian Local Government Communication à la Conférence de l'EIASM, Sienne, Italie, 3-6 septembre.

28. Freeman, E. R. (1984). Strategic Management: A Stakeholder Approach. Pitman, Boston.

29. Germain, C., \& Gates, S. (2007). Le niveau de développement des indicateurs de responsabilité sociale dans les outils de pilotage de contrôle de gestion: une analyse des pratiques des entreprise. Actes $d u$ 28 Congrès de l'AFC, Poitiers.

30. Gibert, P. (1988). Management public, management de la puissance publique. Politique et Management Publics, 4 (2), 89-123.

31. Gray, A., \& Jenkins, B. (1995). From Public Administration to Public Management: Reassessing a Revolution? Public Administration, 73, 75-99.

32. Greiling, D. (2006). Performance Measurement: a Remedy for Increasing the Efficiency of Public Services? International Journal of Productivity and Performance Management, 55 (6), 448-465.

33. Guenoun, M. (2009). Le management e la performance publique locale: etude de l'utilisation des outils de gestion dans deux organisations intercommunales. Thèse pour l'obtention du doctorat ès Sciences de Gestion, Université Paul Cezanne, France, 1-642.

34. Guenoun, M., \& Salery, Y. (2009). La mesure de performance publique locale: variations françaises et anglo-saxonnes. Gestion et Finances Publiques, (11), 893-901.

35. Hockerts, K. (2001). Corporate Sustainability Management, Towards Controlling Corporate Ecological and Social Sustainability. in Proceedings of Greening of Industry Network Conference, January 2124, Bangkok.

36. Hood, C. (1995). The 'New Public Management' in the 1980s: Variations on a Theme. Accounting Organisations and Society, 20, 93109.

37. Hood, C. (2005). Public Management: The Word, the Movement, the Science. in Ferlie E., Lynn J., Laurence C. et Pollitt C. (Eds.). The 
Oxford Handbook of Public Management, Oxford University Press, NY, 7-26.

38. Jensen, M., \& Meckling, W. (1976). Theory of the Firm: Managerial Behaviour, Agency Costs and Ownership structure. Journal of Financial Economics, 3, 305-360.

39. Kaplan, R. S., \& Norton, D. P. (1992). The Balance Scorecard Measures that Drive Performance. Harvard Business Review, 71-79.

40. Kaplan, R. S., \& Norton, D. P. (1996). The Balanced Scorcard: Translating Strategy into Action. Harvard Business School Press, Boston.

41. Kaplan, R. S., \& Norton, D. P. (2001). Comment utiliser le tableau de bord prospectif? Pour créer une organisation orientée stratégie. Éditions d'organisation.

42. Kaufmann, D., Léautier, F., \& Mastruzzi, M. (2005). Governance and the City: an Empirical Exploration into Global Determinants of Urban Performance. World Bank Policy Research Working Paper, (3712), /http://dx.doi.org/10.2139/ssrn.545723.

43. Kuhlmann, S. (2010). New Public Management for the 'Classical Continental European Administration: Modernization at the Local Level in Germany, France and Italy. Public Administration, 88 (4), 1116-1130. doi:10.1111/j.14679299.2010.01869.x.

44. Lorino, P. (2003). Méthodes et pratiques de la performance, le guide du pilotage. $3^{\text {ème }}$ édition, Editions d'Organisation, Paris.

45. Mc Kendrick, J., \& Hastings, M. (2002). The Development of a Performance Framework for Professions Allied to Medicine in Rehabilitation. Communication à l'Annual Congress of the British Accounting Association, St. Helier.

46. Merrien, F. X. (1999). La nouvelle gestion publique : un concept polysémique, Lien Social et Politiques, (41), 96-103.

47. Mongbe, E. (2007). Responsabilisation et transparence, clefs de la santé des finances publiques. Vigie, 10 (3), 4-6.

48. Moullin, M. (2002). Delivering Excellence in Health and Social Care. Open University Press, Buckingham.

49. Moullin, M. (2006). The Design of an Alternative Balanced Scorecard Framework for Public and Voluntary Organisations. Perspectives on Performance, 5 (1).

50. Ndangwa, L., Sonna, F. D., \& Djeumene, P. (2007). Réseau social du dirigeant et performance de la TPE. La Revue Sciences de Gestion, 1 (233), 75-85.

51. Olomo, P. (1987). Comment concilier tradition et modernité dans l'entreprise africaine. Revue Française de Gestion, 91-94. 
52. Osborne, D., \& Gaebler, T. (1992). Reinventing Government: How the Entrepreneurial Spirit is Transforming the Public Sector. Reading, Addison-Wesley.

53. Padovani, E., \& Scorsone, E. (2009). Comparing Local Government's Performance Internationally: A Mission Impossible? International Review of Administrative Sciences, 75 (2), 219-237.

54. Percebois, L. (2006). La promotion de la performance de l'administration publique dans un contexte d'État interventionniste: Le paradoxe scandinave. Colloque International Etat et Régulation Sociale : Comment penser la cohérence de l'intervention publique? 11,12 et 13 sept, 1-19.

55. Poincelot, E., \& Wegmann, G. (2005). Utilisation des critères financiers pour évaluer ou piloter la performance: analyse théorique. Comptabilité-Contrôle-Audit, 11 (2), 109-125.

56. Pollitt, C., \& Bouckaert, G. (2004). Public Management Reform-A Comparative Analysis. Oxford: Oxford University Press.

57. Reynaud, E. (2003). Développement durable et entreprise: vers une relation symbiotique. Journée AIMS, Atelier Développement durable, ESSCA Angers, 15 mai, 1-15.

58. Rochet, C. (2004). Une seule flèche pour deux cibles: le pari ambitieux de la réforme budgétaire en France. Management International, 9 (1), 85-98.

59. Romelaer, P. (2005). L'entretien de recherche, In P. Roussel et F. Wacheux. Management des Ressources Humaines et Sociales: Méthodes de recherche en Sciences Sociales, Bruxelles: Ed. De Boeck.

60. Saulquin, J. F., \& Schier. G. (2005). La RSE comme obligation/occasion de revisiter le concept de performance?, Actes du congrès La Responsabilité Sociale de l'Entreprise : réalité, mythe ou mystification.

61. Sogbossi Bocco, B. (2010). Perception de la notion de performance par les dirigeants de petites entreprises en Afrique. La Revue des Sciences de Gestion, (241), 117-124.

62. Sotamenou, J. (2010). La delegation du service public de gestion des déchets solides en Afrique: à la recherché d'indicateurs de performance. 9th International Conference of territorial intelligence, ENTI, France, 1-13.

63. Supizet, J. (2002). Total Balanced Scorecard, un pilotage aux instruments. L'Informatique Professionnelle, (209), 15-20.

64. Talbot, C. (2003). Les paradoxes de la réforme du management public: l'expérience britannique. Revue Française d'Administration Publique, 2-3 (115-116), 11-24. 
65. Talbot, C. (2005). Performance Management, in Ferlie E., Lynn J., Laurence E., Pollitt C., (Eds.). The Oxford Handbook of Public Management, Oxford University Press, New York, 491-520.

66. Talbot, C. (2006). Performance Regimes and Institutional Context: Comparing Japan, UK and USA. International Symposium on Policy Evaluation, Tokyo, Japon.

67. Van Der Stede, W. A., Chow, C. W., \& Lin, T. W. (2006). Strategy, Choice of Performance Measures and Performance. Behavioral Research in Accounting, 18 (1), 185-205.

68. Veran, L. (2008). La performance, définition légitime et pertinence des indicateurs. in Cadiou C. (Ed.), La performance en management, Presses Universitaires de Rennes, 41-61.

69. Walker, R. M., \& Boyne, G. A. (2009). Introduction: determinants of performance in public organizations. Public Administration, 87 (3), 433-439.

70. Wood, D. J., Mitchell, R. K., Agle, B. R., \& Bryan, L. M. (2018). Stakeholder Identification and Salience after 20 Years: Progress, Problems and Prospects. Business \& Society, 00 (0), 1-50. 